\title{
Automated Identification and Quantification of Signals in Multichannel Immunofluorescence Images
}

\section{The SignalFinder-IF Platform}

\author{
Daniel Barnett, Johnathan Hall, and Brian Haab
}

From the Center for Cancer and Cell Biology, Van Andel Research Institute, Grand Rapids, Michigan

\author{
Accepted for publication \\ March 28, 2019. \\ Address correspondence to \\ Brian Haab, Ph.D., Van Andel \\ Research Institute, 333 Bost- \\ wick NE, Grand Rapids, \\ MI 49503. E-mail: brian. \\ haab@vai.org.
}

\begin{abstract}
Multimarker fluorescence analysis of tissue specimens offers the opportunity to probe the expression levels and locations of multiple markers in a single sample. Software is needed to fully capitalize on the advantages of this technology for sensitive, quantitative, and multiplexed data collection. A major challenge has been the automated identification and quantification of signals. We report on the software SignalFinder-IF, which meets that need. SignalFinder-IF uses a newly developed algorithm called Segment-Fit Thresholding, which showed robust performance for automated signal identification in side-by-side comparisons with several current methods. Two utilities provided with SignalFinder-IF enable downstream analyses. The first allows the quantification and mapping of relationships between an unlimited number of markers through user-defined sequences of AND, OR, and NOT operators. The second produces composite pictures of the signals or colocalization analysis on brightfield hematoxylin and eosin images, which is useful for understanding the morphologies and locations of cells meeting specific marker criteria. SignalFinder-IF enables high-throughput, rigorous analyses of whole-slide, multimarker data, and it promises to open new possibilities in many research and clinical applications. (Am J Pathol 2019, 189: 1402-1412; https://doi.org/10.1016/j.ajpath.2019.03.011)
\end{abstract}

In the analysis of tissue specimens, researchers frequently seek to identify the locations and amounts of specific analytes in the tissue, and then to analyze relationships between the markers and other information. The detection of analytes usually is performed through antibodies that are incubated on the tissue, allowed to bind their targets, and detected by image acquisition. The conventional method of detection is the deposition of colored precipitates produced by the enzymatic conversion of a soluble substrate such as diaminobenzidine to its insoluble form. The enzyme used for the conversion often is horseradish peroxidase, which is attached to a secondary antibody that localizes to the primary antibody. A brightfield image of the tissue typically shows brown staining on top of cells that are visible through hematoxylin and eosin (H\&E) staining. This method has been a workhorse in clinical pathology and research for decades, and it continues to be the primary means of imaging specific proteins in tissue. ${ }^{1-4}$
An increasingly useful and powerful approach for imaging antibody binding is fluorescence. Fluorescence has features that make it preferable to conventional immunohistochemistry in several respects. The signals can be very sensitive-especially with continuing improvements in fluorescence microscopes and scanners - and can be reflective of analyte concentrations over a broad range. ${ }^{5}$ Multiple fluorescence wavelengths may be distinguished from each other with low cross-talk, ${ }^{5}$ which enables the multiplexed detection of multiple analytes in one image, and multiplexing can be greatly expanded through

Supported by National Cancer Institute Early Detection Research Network grant U01CA152653, National Cancer Institute Alliance of Glycobiologists for Cancer Detection grant U01CA168896, and National Institute of Allergy and Infectious Diseases Common Fund Glycoscience Program grant R21AI129872 (B.H.).

D.B. and J.H. contributed equally to this work.

Disclosures: None declared. 
sequential rounds of fluorescence quenching and restaining. ${ }^{6,7}$ Furthermore, fluorescence signals do not obscure the brightfield images of the underlying cells, as can happen with conventional staining.

The features of fluorescence described in the previous paragraph require software for image analysis. A variety of software options currently exist, ${ }^{8,9}$ but a particular challenge has been automation - the ability to accurately identify and quantify signals across all images without user intervention or adjustments. Automation is important for increasing throughput and statistical rigor. It also is important for ensuring that bias from user interpretation is not introduced, and to provide truly objective analyses of large images and data sets. A major challenge is the huge variability among images. Some images have much signal, others have little; some have signalproducing features with unforeseen shapes or sizes; and images can have greatly varying backgrounds. ${ }^{10}$ To automate signal detection, it is necessary to have a fixed basis for extracting meaningful signals that functions across all such characteristics. Basing the signal-detection algorithm on assumed characteristics of the true signals therefore tends to fail for certain images. ${ }^{11}$ Preset parameters may function properly for many images, but they eventually require adjustments of settings by the user. Such a requirement limits throughput and potentially introduces bias, and it necessarily brings some level of subjectivity and arbitrariness to the analysis.

We previously introduced an algorithm that does not rely on assumptions about signal characteristics but instead is based on properties of nonsignal, or background, regions. ${ }^{11}$ It finds nonsignal regions through assumptions about the statistical characteristics of background, which are considerably more predictable than those of signals. The algorithm uses the background regions to properly set thresholds for true signals in that image. Thus, the thresholds are tailored precisely to match each image. Variations between images in intensity, amount, shape, or distribution of signal or background are accounted for properly.

In previous work, ${ }^{11,12}$ initial versions of software were implemented for analyzing multicolor immunofluorescence data and microarray data. The previous software operated well but needed improvements. Because of the computationally intensive nature of the algorithm, the program was too slow to be applied to large images. Large, highresolution images are acquired more regularly with the broader availability of whole-slide fluorescence scanners. In addition, the output formats are varied across the available fluorescence scanners and microscopes that are commercially available, and the range of image formats that could be processed needed to be expanded.

The SignalFinder-IF software package version 4.41 (Haab Laboratory, Van Andel Research Institute, Grand Rapids, MI; https://haablab.vai.org/tools) presented here addresses the limitations described in the previous paragraph and introduces new capabilities for immunofluorescence (IF) analysis. In particular, we aimed to provide a full system of analyzing fluorescence images, which includes downstream analyses and visualization. The identification and quantification of signals often is simply the first step; the information must be evaluated in context among multiple signals and among the cells producing the signals. Our system includes two utilities that use the output of the main SignalFinder-IF program. One is for analyzing and quantifying colocalization among distinct signals, and the other is for preparing composites of the brightfield images of the tissue overlaid with the fluorescence signals. This software package is ready for use on Linux (Linux Foundation, San Francisco, CA), Mac (Apple Inc., Cupertino, CA), and Windows (Microsoft Corporation, Redmond, WA) operating systems.

Here, we present the basic capabilities of the package for multicolor fluorescence image analysis and a head-to-head comparison with several other image analysis methods. The automation ability was compared using only preset parameters without any user review or adjustment. The unique capabilities of the software for quantifying and visualizing relationships between distinct markers also have been shown.

\section{Materials and Methods}

\section{Software Development and Data Analysis}

The software was developed and tested using MATLAB (Mathworks Inc., Natick, MA), Java (Oracle Corporation, Redwood Shores, CA), and C++ (International Organization for Standardization, Geneva, Switzerland). Microsoft Excel (Microsoft Corporation) was used for analyzing numeric output, GraphPad Prism (GraphPad Software Inc., San Diego, CA) for the preparation of graphs, and Canvas XIV (Canvas GFX, Plantation, FL) for the preparation of figures.

\section{Immunofluorescence Data and Image Processing}

The immunofluorescence data were acquired previously, ${ }^{12}$ but are summarized briefly here. Immunofluorescence was performed on $5-\mu \mathrm{m}$-thick sections cut from formalin-fixed, paraffin-embedded blocks. Two primary antibodies were labeled with Sulfo-Cyanine5 NHS ester (13,320; Lumiprobe Corporation, Hunt Valley, MD) and Sulfo-Cyanine3 NHS ester (11320; Lumiprobe Corporation) according to the supplier's protocol. Each round of immunofluorescence used two different antibodies and nuclear staining with Hoechst 33258. After staining, the slides were scanned using a scanningfluorescence microscope (Vectra; PerkinElmer, Waltham, MA). The microscope collected 35 images at each field-ofview, each image at a different emission wavelength. The fluorescence was quenched using $6 \% \mathrm{H}_{2} \mathrm{O}_{2}$ in $250 \mathrm{mmol} / \mathrm{L}$ sodium bicarbonate ( $\mathrm{pH} 9.5$ to 10 ), and another round of immunofluorescence was performed using two different antibodies. The subsequent incubations and scanning steps were as described earlier in this section. The H\&E staining followed a standard protocol. 


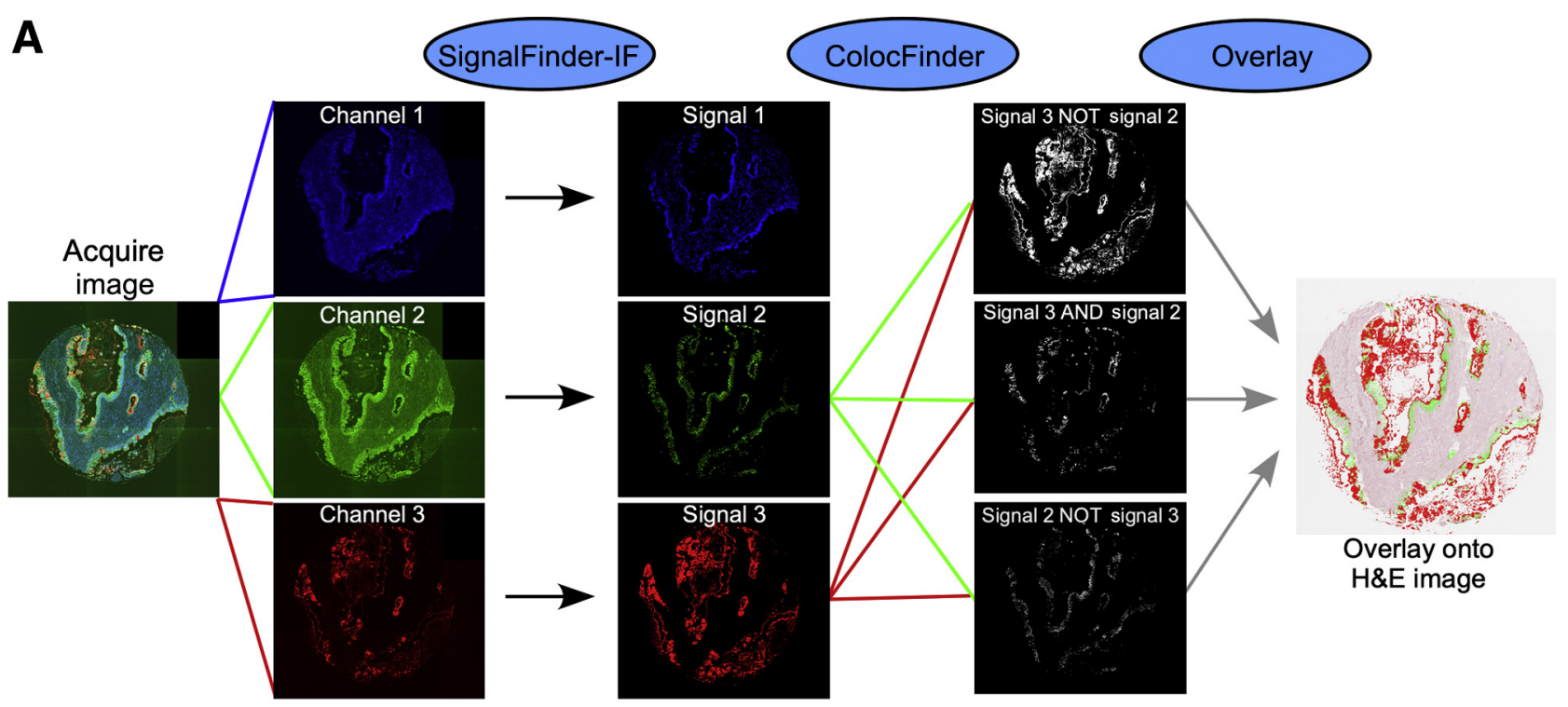

B

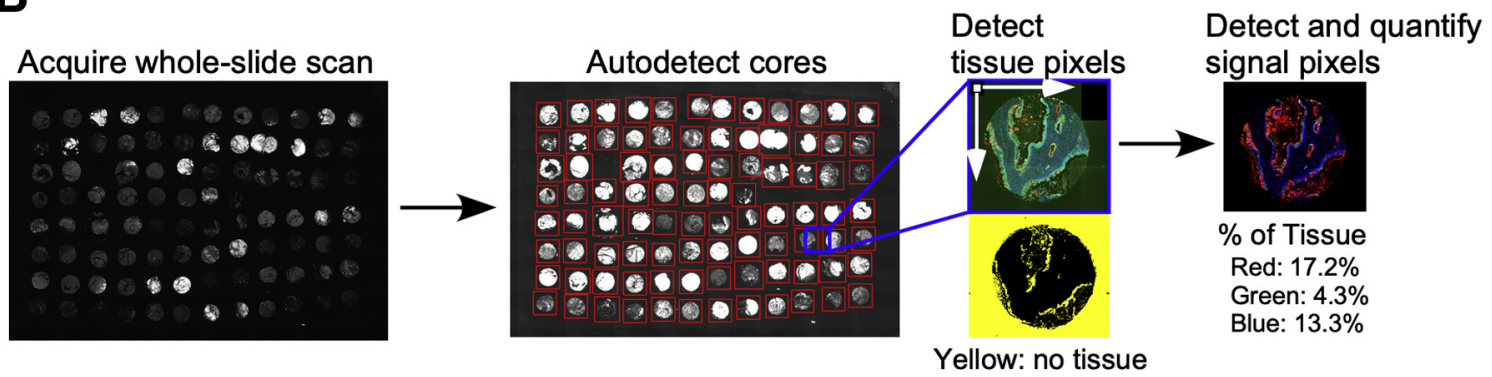

Figure 1 The SignalFinder-IF system. A: Package components. The package includes the core SignalFinder-IF program and the utilities Colocfinder and overlay. SignalFinder-IF separately analyzes individual color channels specified by the user. It identifies and quantifies the signal and produces a map of the signal pixels. ColocFinder identifies regions of the image fulfilling criteria defined by the user. It quantifies the amount of tissue fulfilling the terms and produces a map of the output pixels. The overlay program aligns the output from SignalFinder-IF or ColocFinder to a brightfield image and produces a composite, overlaid image using color schemes set by the user. B: Automated analysis of tissue microarray images. SignalFinder-IF detects the individual cores, quantifies signal in each color channel from each core, and assembles the data with incorporation of any metadata provided by the user. Original magnification: $\times 20(A) ; \times 4$ (B, left two images); $\times 20$ (B, right two images). H\&E, hematoxylin and eosin.

From the 35 images captured for each region, the 3 that corresponded to the emission maxima of Hoechst 33258, Cy3, and Cy5 were selected. For each image, SignalFinder-IF generated a map of the pixel locations containing signal and computed various values for the output report, such as the percentage of tissue-containing pixels that had signal.

\section{Results}

Flow of SignalFinder-IF Processing

The overall analysis system includes the core SignalFinderIF program as well as two utilities for analyzing the output of SignalFinder-IF: ColocFinder and Overlay (Figure 1A).

Table 1 Markers Analyzed in the Tissue Microarrays

\begin{tabular}{|c|c|c|c|c|c|c|c|}
\hline TMA name & Cases & $\begin{array}{l}\text { Marker } 1 \\
\text { (blue) }\end{array}$ & $\begin{array}{l}\text { Marker } 2 \\
\text { (green) }\end{array}$ & $\begin{array}{l}\text { Marker } 3 \\
\text { (red) }\end{array}$ & $\begin{array}{l}\text { Marker } 4 \\
\text { (blue) }\end{array}$ & $\begin{array}{l}\text { Marker } 5 \\
\text { (green) }\end{array}$ & $\begin{array}{l}\text { Marker } 6 \\
\text { (red) }\end{array}$ \\
\hline TMA2: human primary tumors & 27 & Hoechst & E-cadherin & CA19-9 & Hoechst & Vimentin & sTRA \\
\hline TMA5: human primary tumors & 24 & Hoechst & MUC5AC & CA19-9 & Hoechst & $\beta$-catenin & sTRA \\
\hline TMA6: human primary tumors & 26 & Hoechst & MUC5AC & CA19-9 & Hoechst & $\beta$-catenin & sTRA \\
\hline TMA68: cell line xenografts & 10 & Hoechst & MUC5AC & CA19-9 & Hoechst & $\beta$-catenin & sTRA \\
\hline TMA69: patient-derived xenografts & 14 & Hoechst & MUC5AC & CA19-9 & Hoechst & $\beta$-catenin & sTRA \\
\hline
\end{tabular}

Images were selected from 11 cores across the TMAs, using six images per core (one image for each marker), for a total of 66 images used in the method comparison.

CA19-9, carbohydrate antigen 19-9; MUC5AC, mucin 5 subtypes A and C; sTRA, sialylated tumor-related antigen; TMA, tissue microarray. 

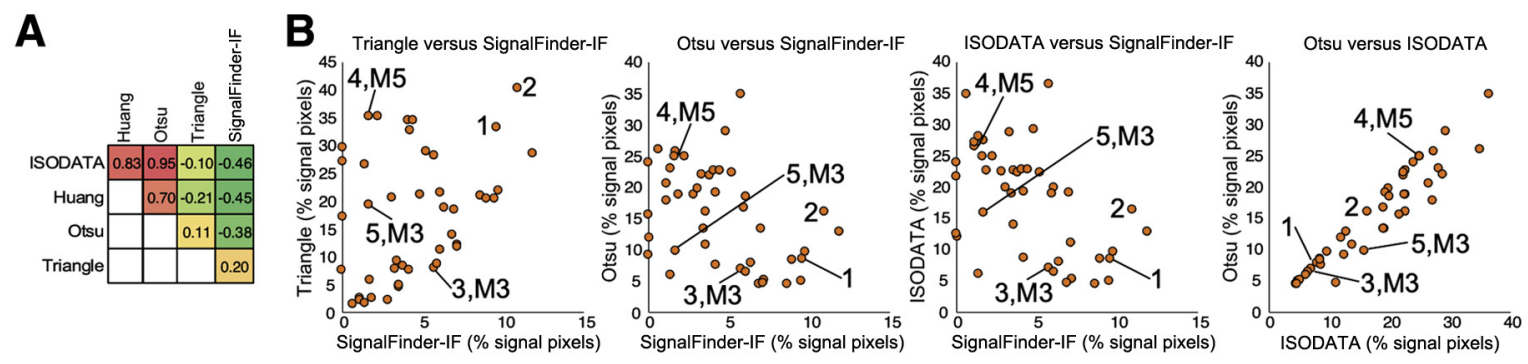

C

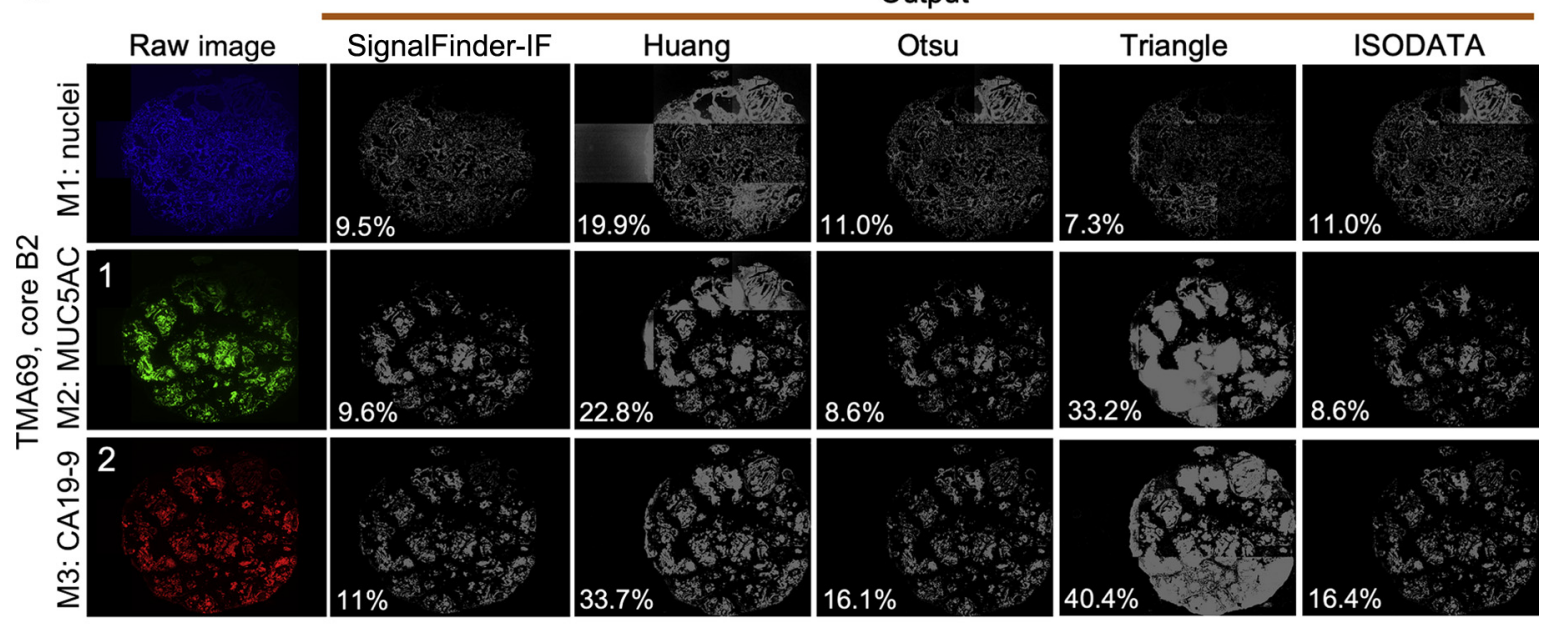

D
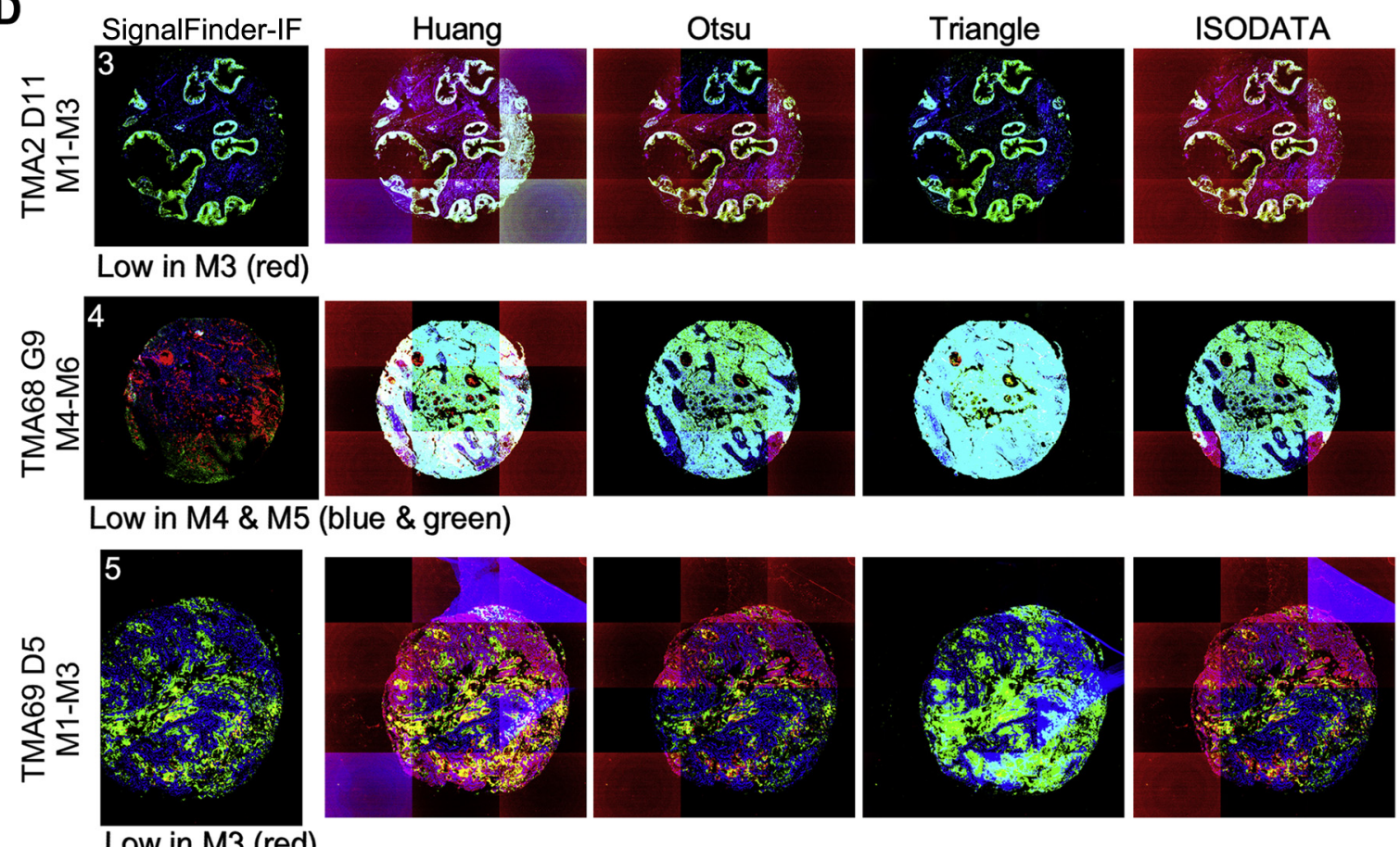

Figure 2 Automated image analysis of tissue microarray (TMA) data. A: Correlations between methods. Eleven cores were selected across the five TMAs and each of the six markers was analyzed in each core (Table 1), for a total of 66 images analyzed. The matrix provides the Pearson correlation coefficients, comparing the percentage signal found by each method across the 66 images. B: Variability in individual results. The plots present the quantified output from the 11 cores and 4 markers (excluding the nuclei-staining data). Each point is the percentage signal from one marker and one core. The numbers in B correspond to the images in C and D. C: Comparisons of single-channel outputs. The left column shows the raw-fluorescence data, and the next columns show the pixels identified as signal by each method in each color channel, with the percentage of pixels that are positive. D: Comparisons over a range of image characteristics. Each image shows the pixels identified as signal in each of the three color channels, combined into one image. The colors represent mixing of the blue, green, and red channels. Original magnification, $\times 20$. M1-M6, markers 1-6. CA19-9, carbohydrate antigen 19-9. 


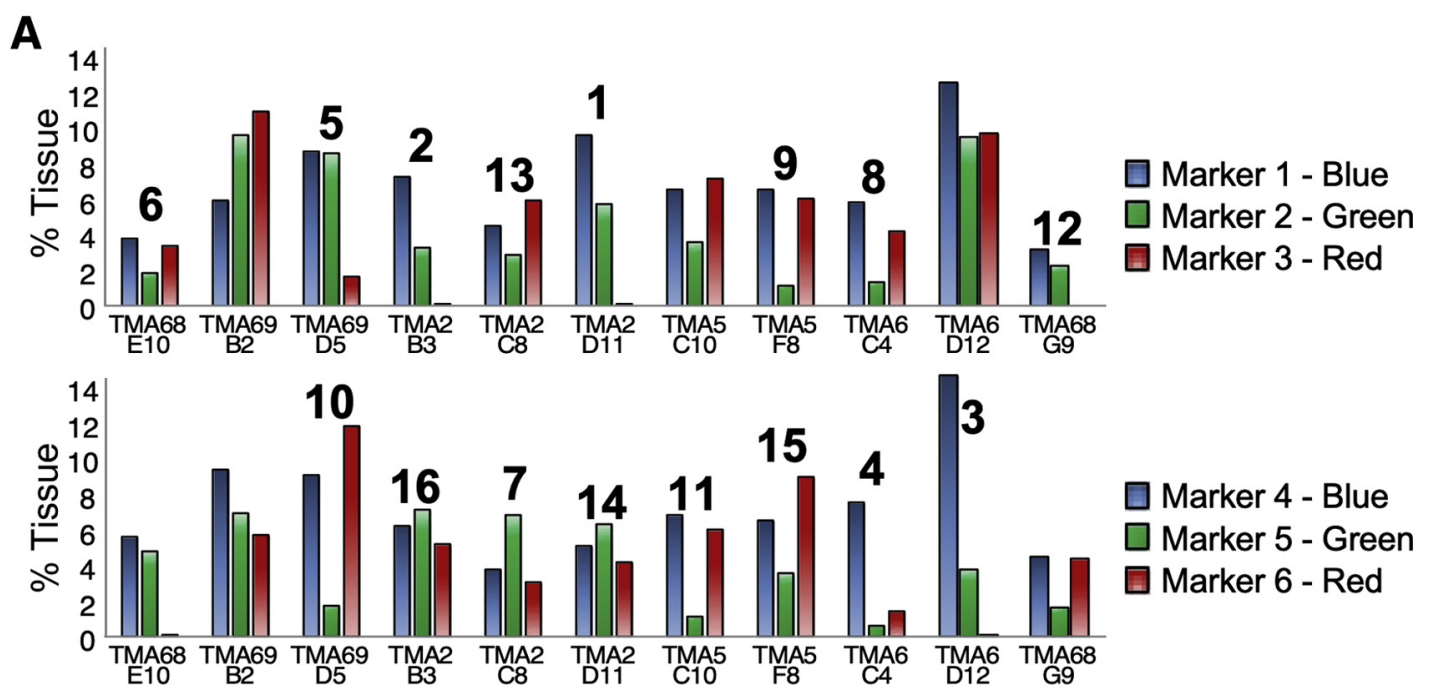

B
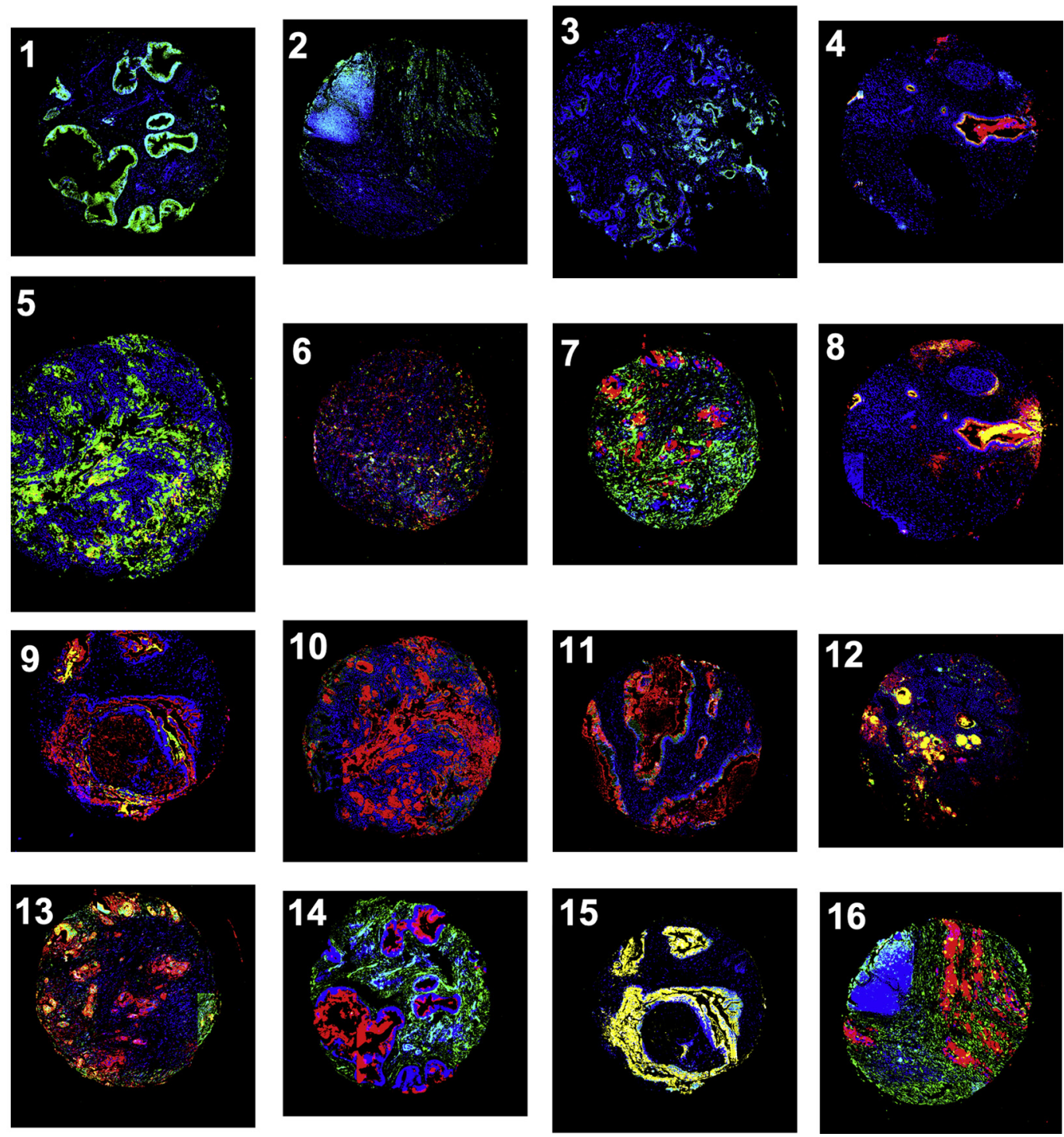

Figure 3 Exploration of consistency. A: Quantified output from the 66 images. The column graphs present the quantified output from the 11 cores and six markers, showing a range of high, medium, and low values for each color. The numbers above the columns in $\mathbf{A}$ correspond to the images in $\mathbf{B}$. B: Representative images. Each image is the combined output from the analysis of the blue, green, and red colors from one of the rounds, either markers 1 to 3 or markers 4 to 6 . The colors represent mixing of the three colors. Original magnification, $\times 20$. 


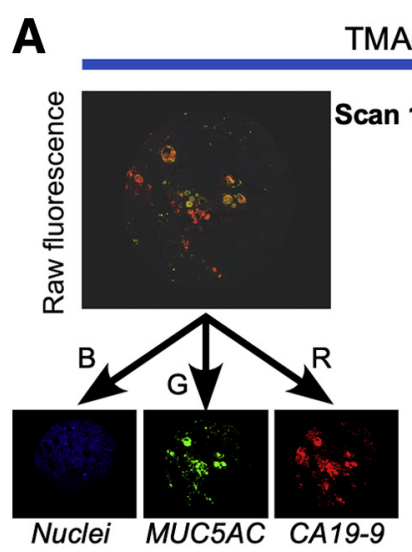

A68-G9

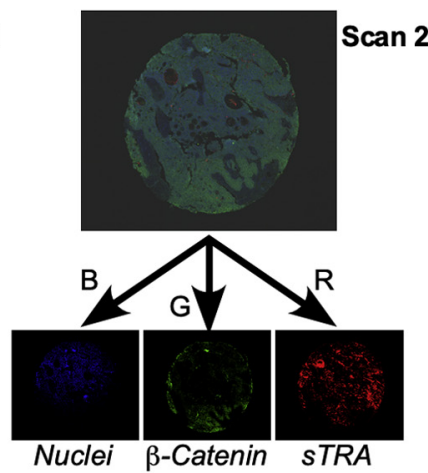

B CA19-9 NOT STRA
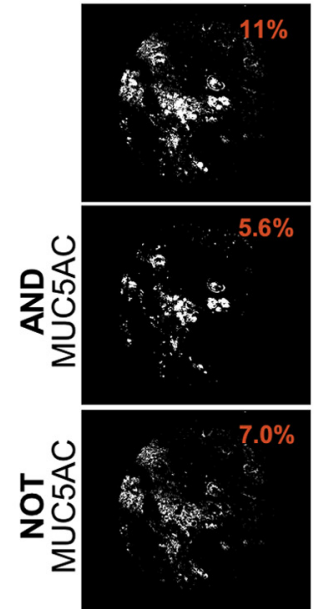

CA19-9 AND STRA
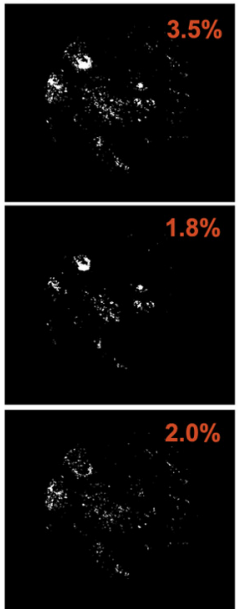

STRA NOT CA19-9
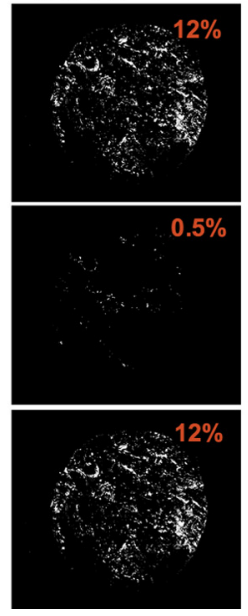

TMA69-B2
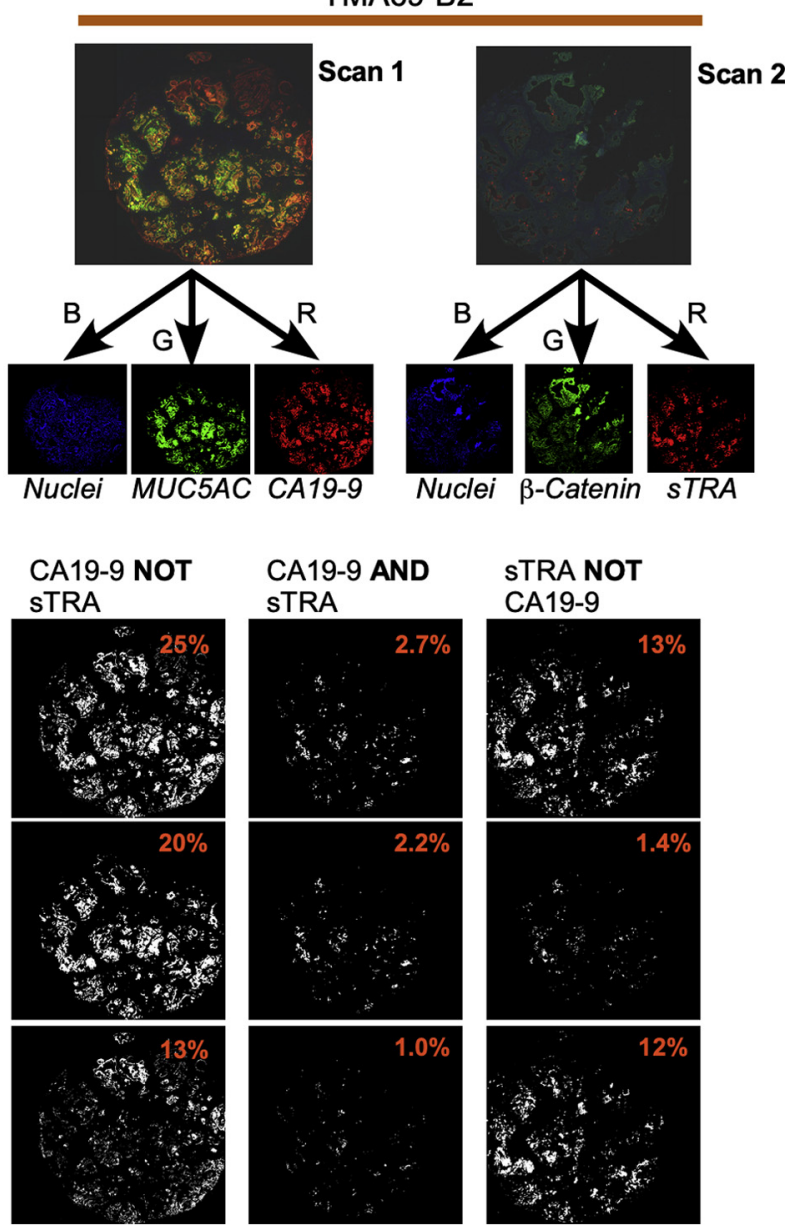

STRA NOT

CA19-9
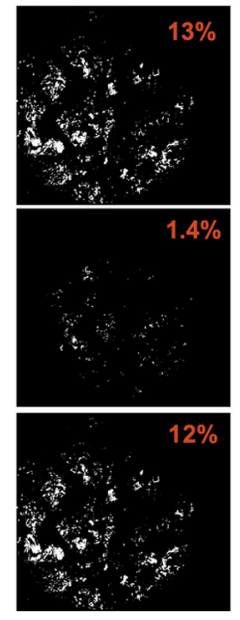

Figure 4 Exploring relationships between markers. A: Primary quantification of each marker. Three color channels were acquired in two separate scans, resulting in six markers. The automated signal-finding algorithm identified the signals in the blue, green, and red channels. B: Quantifying various relationships. The colocalization utility provides the mapping of user-defined relationships between any number of markers. The example shows the combined and exclusive expression of carbohydrate antigen 19-9 (CA19-9) and sialylated tumor-related antigen (sTRA), followed by adding combinations with mucin 5 , subtypes $A$ and $C$ (MUC5AC). Original magnification, $\times 20$.

The SignalFinder-IF program retrieves the color channels defined by the user, and then analyzes each channel separately to find the background and signal pixels. ColocFinder is for analyzing the relationships between the signals from individual channels, and Overlay is for producing composites of the individual or colocalized signals overlaid on the brightfield images.

The package is well suited to analyze tissue microarrays (TMAs) or whole slides with multiple, separate pieces of tissue (Figure 1B). TMAs are useful for acquiring data on many tissue specimens on a single slide, but the amount of information from one experiment can be overwhelming if processed manually. SignalFinder-IF detects the tissue cores automatically, or with assistance from the user if necessary, and analyzes the image data for each tissue core independently. For proper normalization of the extent of signal, SignalFinder-IF determines the amount of tissue present in each images. This step is important because the image for a core can include regions with no tissue, which affects the interpretation of the extent of staining. Sometimes only a minimal portion of the image contains tissue, for example, if the specimen is fragmented or partly washed off. SignalFinder-IF quantifies the number of pixels that contain tissue for each core, and then it divides the number of signal pixels by the number of tissue pixels (Figure 1B).

The final output is based on either the extent of signal-the amount of the image containing signal—or the intensity of the signal. The threshold for identifying signal pixels is based on the following formula: $\mathrm{T}=$ mean $+\mathrm{M} *$ $\mathrm{SD}$, where $\mathrm{T}$ is the pixel threshold, mean and $\mathrm{SD}$ are the mean and SD of the background, respectively, and $\mathrm{M}$ is a multiplier specified by the user (default $=3$ ). The primary output is the percentage of pixels that surpass the threshold and are counted as signal pixels (filters are in place to prevent spikes from being counted as signals), and the secondary output is the average intensity of the signal pixels. The software repeats these two outputs for as many thresholds as specified by the user. As a standard output, the 
A

Whole-core overlay images
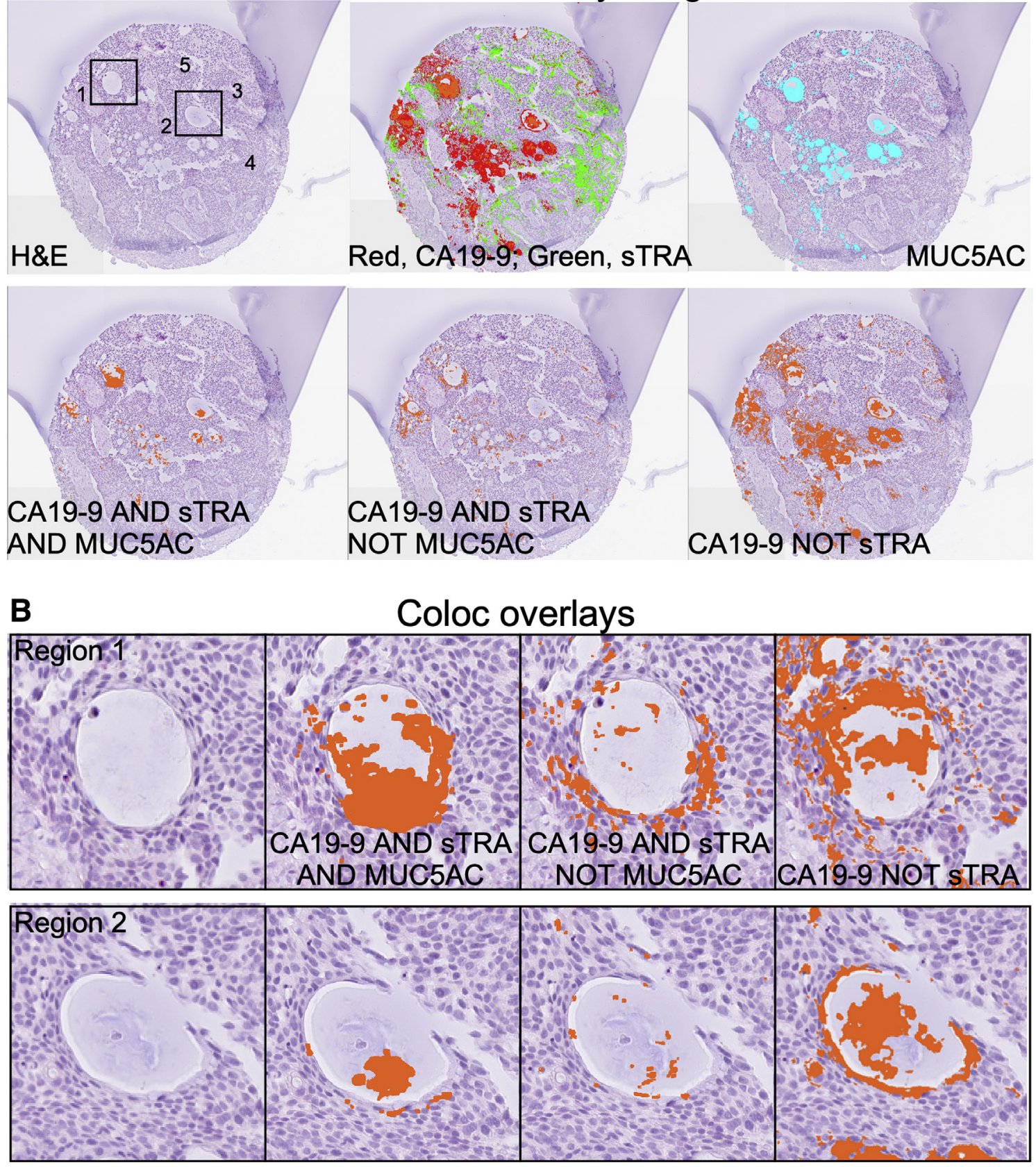

C SignalFinder-IF overlays

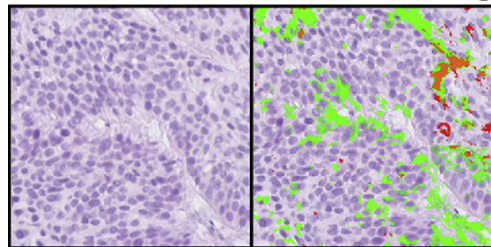

Region 3

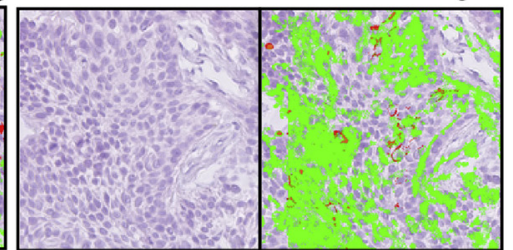

Region 4

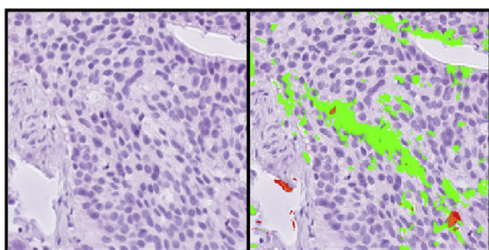

Region 5

\section{Red, CA19-9; Green, sTRA}

Figure 5 Composite images. A: Whole-core overlay images. Either the SignalFinder-IF output or the ColocFinder output can be overlaid on the whole-core hematoxylin and eosin (H\&E) image. B: Overlay images of the output from ColocFinder. Zooms of specific regions provide detailed views of the cells fulfilling various relationships between the markers. C: Overlay images of the output from SignalFinder-IF. Detailed views show the unique morphologies of the cells expressing primarily sialylated tumor-related antigen (sTRA). The region numbers correspond to those in A. Original magnification, $\times 20$. CA19-9, carbohydrate antigen 19-9; MUC5AC, mucin 5, subtypes A and C. 
use of percentages at various thresholds is preferable because the raw intensities are subject to high differences between experiments based on varying experimental factors, whereas the percentages are less sensitive to those factors. This approach mirrors typical analyses in histopathology, in which the analyst would estimate the extent of staining at various intensity levels.

\section{Accuracy in Automated Analysis}

The challenge in automated image analysis is being able to handle a wide range of image characteristics accurately, without user review and adjustments of settings. TMA data provide a good test of this capability because the individual tissue specimens have many varied characteristics. Immunofluorescence data were used from five different TMAs, selecting 11 different 1-mm tissue cores. The acquisition of the images was performed in earlier work, ${ }^{12}$ and it involved the multiplexed detection of three color channels (blue, green, and red) in two separate rounds. In each round, the blue channel was a nuclear stain, and the green and red channels were for monoclonal antibodies labeled with $\mathrm{Cy} 3$ or Cy5, targeting four different markers (Table 1). The method of using multiple rounds of staining enables multiplexed immunofluorescence using a limited number of distinct fluorophores. ${ }^{6}$ Sialylated tumor-related antigen (sTRA), a glycan that was identified previously as a strong serologic biomarker of pancreatic cancer, was studied. ${ }^{13,14}$ It performed as well as the current best serologic biomarker for pancreatic cancer, carbohydrate antigen 19-9 (CA19-9), which also detects a glycan, and it was increased in approximately half of the patients with low CA19-9, indicating independent regulation. In addition to data for the two glycans, data also were acquired for the proteins mucin 5 , subtypes A and C (MUC5AC), $\beta$-catenin, vimentin, and E-cadherin (Table 1). Between the four markers and the two rounds of nuclear staining (six images per tissue) for the 11 different cores, 66 different images were analyzed for this study (Figure 2A).

SignalFinder-IF was compared with four commonly used methods for image analysis of immunofluorescence data. The comparison methods were as follows: i) the Iterative Self-Organizing Data Analysis Technique (ISODATA) method $^{15}$; ii) the Huang and Wang ${ }^{16}$ threshold; iii) the Otsu ${ }^{17}$ method; and iv) the triangle threshold. ${ }^{18,19}$ For each of the five algorithms, the settings provided by the software for automated image analysis were used. SignalFinder-IF is designed to handle multiple tissue specimens from TMAs or similar data, but a custom script was needed to process the images using the other methods.

For each of the methods, the quantification of the amount of signal detected across the 66 images was assembled. The quantification used was the percentage of the image in which signal was detected, based on the threshold and criteria set automatically by each method. The pairwise correlations between the methods in their output values across the 66 images then were examined (Figure 2A). The ISODATA, Huang and Wang, ${ }^{16}$ and Otsu ${ }^{17}$ methods correlated well with each other, whereas SignalFinder-IF was different from the others but was related most closely to the triangle method (Figure 2A). The quantified data generally were higher for the comparison methods relative to SignalFinder-IF, with some images showing major differences in quantification between the methods (Figure 2B).

Based on the comparisons of the quantifications, some of the images with similar or divergent results were selected for visual inspection (Figure 2C). Each core image comprises the tiled high-magnification fields from the scanning microscope, with a total of eight to nine fields per core. SignalFinder-IF showed good consistency across the fields and colors in finding signal pixels in agreement with the raw fluorescence of each color. Each of the comparison methods showed comparable results in selected colors and fields, but each also showed locations or entire images in which signal identification was too permissive or too stringent. Images with diverse quantifications from three additional cores (Figure 2B), in which the outputs from the three colors were assembled, showed similar results (Figure 2D).

The individual output data across additional cores that were greatly different from one another in their quantifications were examined next. The summary output from SignalFinder-IF over the 66 images showed that each core had a unique pattern of results from the various markers (Figure 3A). Various cores were selected and the assembled three-color output from each was examined (Figure 3B). In each case, the output from each color channel provided a viable identification of presumptive signals, without spurious identifications outside of the tissue areas, and with good consistency between the tiled fields. This result shows good consistency in automated analysis across many highly diverse images.

\section{Analysis of Relationships between Markers}

A valuable feature of fluorescence in comparison with visible stains is the multiplexing capability. Antibodies can be labeled with different dyes to detect distinct targets that are used routinely to probe three or four targets in one run. In addition, using the multiround method shown here, researchers have acquired data from dozens of markers without evidence of interference or cross-talk between markers. ${ }^{6,20}$ As a result of this multiplexing capability, an important use of immunofluorescence experiments is to detect colocalization of fluorescent signals from distinct probes. $^{21}$

Software was designed to quantify exclusive expression as well as colocalization (Figure 4A). The ColocFinder utility allows the user to build expressions of AND, OR, and NOT between scans, and it then quantifies the percentage of pixels that fulfill the expression. The AND operator requires signal pixels to be present in both scans, the OR operator requires pixels to be present in either scan, and the NOT 
operator requires pixels to be present in the first but not the second scan.

The signals were examined from CA19-9, sTRA, CA19-9 in the absence of sTRA (CA19-9 NOT sTRA), sTRA in the absence of CA19-9 (sTRA NOT CA19-9), and the expression of both CA19-9 and sTRA (CA19-9 AND sTRA) colocalized (Figure 4B). The program first registers the images to be compared with each other containing the results to be evaluated. It then scans a sliding box of userdefined size (ie, the colocalization radius or desired proximity to evaluate for two or more markers) across the data and evaluates the pixels in each segment according to the user-defined relationship. If a pixel meets the criteria in a minimum number of the segments, it is counted as positive, otherwise it is negative. The output is the percentage of pixels relative to the number of pixels in the image containing tissue that fulfill the criteria specified by the user.

The three relationships described in the previous paragraph could be examined relative to another marker such as MUC5AC (Figure 4B). The pixel maps and quantification show that most of the MUC5AC is colocalized with CA19-9 in the absence of sTRA, but some is colocalized with both. Thus, complex relationships among multiple markers in their combined and exclusive expression can be visualized and quantified by this system. ColocFinder uses a novel algorithm that does not require complete overlap in pixels, so the various outputs are not mutually exclusive. The flexibility in finding regions fulfilling the search terms is a good option when markers would be expected to be near one another but not necessarily overlapping. Examples would be two extracellular markers, or a membranous and extracellular marker, or two markers in cells or subcellular compartments with unpredictable shapes.

\section{Production of Composite Images}

Another advantage of fluorescence is that the signals do not hinder the acquisition of a high-quality brightfield image of the underlying cells. Obtaining a good picture of the underlying cells can be important for determining the types and morphologies of the cells producing certain markers. To facilitate this type of analysis, a utility that uses the output of the signal-finding algorithm or the colocalization analysis to produce images of the fluorescence or colocalization data overlaid on the brightfield image was developed. The program registers the SignalFinder-IF or ColocFinder output to the brightfield image using the nuclei because the nuclei provide the most consistent signal from the Hoechst stain in every round. It extracts the appropriate color range for nuclei in the H\&E image, and then registers the output data using the nuclei signal from the scan. The registration can be adjusted manually if necessary. The program then generates images of the output overlaid on the $\mathrm{H} \& \mathrm{E}$ picture, using a color scheme set by the user.

Views of the whole tissue or core can provide information on the locations of features of interest (Figure 5A). Images zoomed into specific regions can provide information on the morphologies of cells with particular characteristics. For example, the epithelial layer of a gland expressing both CA19-9 and sTRA and secreting MUC5AC into the lumen has cells with little cytoplasm, and the epithelium of another gland producing almost exclusively CA19-9 has more columnar cells (Figure 5B). The cells expressing primary sTRA, and not CA19-9, form small, ill-defined glandular features (Figure $5 \mathrm{C}$ ). The ability to view the original H\&Estained images with the composite images side-by-side can help to identify such features.

\section{Discussion}

The increasing availability and quality of whole-slide fluorescence scanning has resulted in increased adoption of this powerful technology. Herein, we present software that meets the demand for automated signal detection and flexible downstream analyses. The method used here allowed identification and analysis of signals from multiple markers across many images with widely varying characteristics, with full automation of the primary image analysis. Such analyses would be extremely time consuming if performed manually. Furthermore, manual analyses are subject to bias from the user, whereas automated analyses are inherently objective. Objectively quantified values are useful in downstream studies of marker expression in tissue, for example, in biomarker studies using TMAs.

The key to the robust performance of SignalFinder-IF in automated analysis is the algorithm of statistically identifying the background characteristics for each image individually, so that thresholds can be tailored to each image. Each of the comparison methods presented here performed well for some images but had inaccurate signal finding in other cases. The algorithms used by the comparison methods evaluate aspects of the distributions of the signal intensities, with the goal of separating background from signal. The ISODATA clustering algorithm uses iterative testing to find the greatest Euclidean distance between signal and background clusters. The Huang and Wang ${ }^{16}$ fuzzy threshold method steps through thresholds and uses an optimization function across thresholds to determine the true valley between the background and signal peaks. The method of Otsu ${ }^{17}$ takes the maximum point of intraclass variance and steps through thresholds until it finds maximum variance between the two classes on either side of the threshold. The triangle method draws a line from the peak of the histogram to the tail of the peak and finds the maximum distance from the line to the curve, which sets the threshold at the inflection point at the end of the primary peak. It then takes anything above that as signal. SignalFinder-IF, in contrast, first finds the background pixels, and then finds signal pixels based on thresholds derived from the background pixels. It also has a method to disallow spurious spikes in the data to be counted as signal pixels. Thus, SignalFinder-IF, although more processing- 
intensive than other methods, provides better adjustments to background variation. The many images presented here highlight the advantage of not depending on a certain amount, shape, or distribution of the signal; if no signal is present, it will detect no signal.

The novel component provided by the ColocFinder utility is the exploration of complex relationships between markers. The currently available software packages typically quantify simple co-occurrence between two markers. For example, a frequently used method introduced by Manders et $\mathrm{al}^{22}$ uses correlations between color channels to calculate average overlap in signals. This information is useful, but additional relationships would be important to probe. Of particular interest is the possibility that the exclusive expression of a particular marker, that is, the presence of one marker in the absence of another, could be a marker of phenotype. Given that certain cell types or tissue phenotypes are identified by the absence and presence of certain markers, researchers should find utility in the quantification of both the exclusive and the concurrent expression of various markers.

To further complement this information, the Overlay utility allows users to identify by visual analysis which cells meet various marker-expression characteristics. Thus, between the core SignalFinder-IF program and the ColocFinder and Overlay utilities, one may integrate quantitative analysis with visual-based analysis. The quantification provided by SignalFinder-IF can mirror common practice in pathology, in which the pathologist examines the percentage of an image with high-intensity signal and the percentage with low-intensity signal. Likewise, SignalFinder-IF scores the amount of the image containing signal-given as the percentage of pixels that are counted as signal pixels - and the average intensity of the signal pixels at multiple thresholds that can be set by the user. This information can be used in subsequent statistical evaluations or in further processing through ColocFinder and Overlay.

The SignalFinder-IF platform has limitations not found in other systems. Because it does not locate features based on shape or size, it cannot be used to quantify subcellular components or cell counts using such features. The extensive computation involved in analyzing the statistical characteristics of the image require more computer memory and faster processors than required for other methods. The time needed for the program to analyze the images also could be greater than needed for other programs. Thus, various image-analysis systems could be used in conjunction with each other to provide complementary capabilities.

SignalFinder-IF may be useful for a wide range of research and technological applications, such as in the analysis of immunofluorescence signals from cohorts of patients. ${ }^{23}$ In clinical applications, automated image analysis could help to remove interoperator variability or to pick out rare or subtle features. If the user requires precise and objective quantification, or analysis of signals that are difficult to locate by eye, or the analysis of many data sets, automated quantification is preferable. ${ }^{8,9}$

\section{References}

1. McCourt CM, Boyle D, James J, Salto-Tellez M: Immunohistochemistry in the era of personalised medicine. J Clin Pathol 2013, 66:58-61

2. Hamilton PW, Bankhead P, Wang Y, Hutchinson R, Kieran D, McArt DG, James J, Salto-Tellez M: Digital pathology and image analysis in tissue biomarker research. Methods 2014, 70:59-73

3. Salto-Tellez M: Diagnostic molecular cytopathology - a further decade of progress. Cytopathology 2015, 26:269-270

4. Jones JL, Oien KA, Lee JL, Salto-Tellez M: Morphomolecular pathology: setting the framework for a new generation of pathologists. $\mathrm{Br}$ J Cancer 2017, 117:1581-1582

5. Carvajal-Hausdorf DE, Schalper KA, Neumeister VM, Rimm DL: Quantitative measurement of cancer tissue biomarkers in the lab and in the clinic. Lab Invest 2015, 95:385-396

6. Gerdes MJ, Sevinsky CJ, Sood A, Adak S, Bello MO, Bordwell A, Can A, Corwin A, Dinn S, Filkins RJ, Hollman D, Kamath V, Kaanumalle S, Kenny K, Larsen M, Lazare M, Li Q, Lowes C, McCulloch CC, McDonough E, Montalto MC, Pang Z, Rittscher J, Santamaria-Pang A, Sarachan BD, Seel ML, Seppo A, Shaikh K, Sui Y, Zhang J, Ginty F: Highly multiplexed single-cell analysis of formalin-fixed, paraffin-embedded cancer tissue. Proc Natl Acad Sci U S A 2013, 110:11982-11987

7. Lin JR, Fallahi-Sichani M, Sorger PK: Highly multiplexed imaging of single cells using a high-throughput cyclic immunofluorescence method. Nat Commun 2015, 6:8390

8. Eliceiri KW, Berthold MR, Goldberg IG, Ibanez L, Manjunath BS, Martone ME, Murphy RF, Peng H, Plant AL, Roysam B, Stuurman N, Swedlow JR, Tomancak P, Carpenter AE: Biological imaging software tools. Nat Methods 2012, 9:697-710

9. Niederlein A, Meyenhofer F, White D, Bickle M: Image analysis in high-content screening. Comb Chem High Throughput Screen 2009 12:899-907

10. Choudhury KR, Yagle KJ, Swanson PE, Krohn KA, Rajendran JG: A robust automated measure of average antibody staining in immunohistochemistry images. J Histochem Cytochem 2010, 58: 95- 107

11. Ensink E, Sinha J, Sinha A, Tang H, Calderone HM, Hostetter G, Winter J, Cherba D, Brand RE, Allen PJ, Sempere LF, Haab BB: Segment and fit thresholding: a new method for image analysis applied to microarray and immunofluorescence data. Anal Chem 2015, 87: 9715-9721

12. Barnett D, Liu Y, Partyka K, Huang Y, Tang H, Hostetter G, Brand RE, Singhi AD, Drake RR, Haab BB: The CA19-9 and sialylTRA antigens define separate subpopulations of pancreatic cancer cells. Sci Rep 2017, 7:4020

13. Tang H, Partyka K, Hsueh P, Sinha JY, Kletter D, Zeh H, Huang Y, Brand RE, Haab BB: Glycans related to the CA19-9 antigen are elevated in distinct subsets of pancreatic cancers and improve diagnostic accuracy over CA19-9. Cell Mol Gastroenterol Hepatol 2016, 2: 201-221.e15

14. Staal B, Liu Y, Barnett D, Hsueh P, He Z, Gao CF, Partyka K, Hurd MW, Singhi AD, Drake RR, Huang Y, Maitra A, Brand RE, Haab BB: The sTRA plasma biomarker: blinded validation of improved accuracy over CA19-9 in pancreatic cancer diagnosis. Clin Cancer Res 2019, 29: 2745-2754

15. Ridler TW, Calvard S: Picture thresholding using an iterative selection method. IEEE Trans Syst Man Cybernetics 1978, 8:630-632

16. Huang LK, Wang MJJ: Image thresholding by minimizing the measures of fuzziness. Pattern Recognition 1995, 28:41-51

17. Otsu N: A threshold selection method from gray-level histograms. IEEE Trans Sys Man Cyber 1979, 9:62-66 
18. Zack GW, Rogers WE, Latt SA: Automatic measurement of sister chromatid exchange frequency. J Histochem Cytochem 1977, 25:741-753

19. Rosin PL: Unimodal thresholding. Pattern Recognition 2001, 34: 2083-2096

20. Riordan DP, Varma S, West RB, Brown PO: Automated analysis and classification of histological tissue features by multi-dimensional microscopic molecular profiling. PLoS One 2015, 10:e0128975
21. Bolte S, Cordelieres FP: A guided tour into subcellular colocalization analysis in light microscopy. J Microsc 2006, 224:213-232

22. Manders EMM, Verbeek FJ, Aten JA: Measurement of co-localization of objects in dual-colour confocal images. J Microsc 1993, 169:375-382

23. Kallioniemi OP, Wagner U, Kononen J, Sauter G: Tissue microarray technology for high-throughput molecular profiling of cancer. Hum Mol Genet 2001, 10:657-662 